\title{
Changes in kidney function among patients undergoing transcatheter aortic valve replacement
}

\author{
Charat Thongprayoon ${ }^{1}$, Wisit Cheungpasitporn ${ }^{1}$, Wonngarm Kittanamongkolchai ${ }^{1}$, Narat Srivali ${ }^{2}$, Kevin \\ L Greason $^{3}$, Kianoush Kashani ${ }^{1,2^{*}}$
}

${ }^{1}$ Division of Nephrology and Hypertension, Mayo Clinic, Rochester, MN, USA

${ }^{2}$ Division of Pulmonary and Critical Care Medicine, Mayo Clinic, Rochester, MN, USA

${ }^{3}$ Division of Cardiovascular Surgery, Department of Medicine, Mayo Clinic, Rochester, MN, USA

\section{A R T I C L E I N F O}

Article Type:

Original

\section{Article History:}

Received: 28 December 2016

Accepted: 8 February 2017

Published online: 22 February 2017

\section{Keywords:}

Acute kidney injury

Kidney function

Glomerular filtration rate

Transcatheter aortic valve replacement

\begin{abstract}
A B S T R A C T
Introduction: The patients selected for transcatheter aortic valve replacement (TAVR) usually have a high prevalence of chronic kidney disease (CKD). Little is known regarding the impact of TAVR on changes in renal function.

Objectives: This study aimed to assess the change in estimated glomerular filtration rate (eGFR) after TAVR.

Patients and Methods: Adult patients with aortic stenosis (AS) who underwent TAVR between January 2008 and June 2014, at Mayo Clinic, Rochester, MN. Changes in renal function during six months follow-up were evaluated.

Results: Of 386 patients undergoing TAVR, 106 (28\%) developed acute kidney injury (AKI). There was significant reduction in eGFR at the hospital discharge and at 6 months post-TAVR in AKI patients in comparison with non-AKI individuals, (mean differences -7.1; 95\% CI $-9.8,-4.3 \mathrm{~mL} / \mathrm{min} / 1.73 \mathrm{~m}^{2}, P<0.001$ and $-4.2 ; 95 \% \mathrm{CI}-7.1,-1.3 \mathrm{~mL} / \mathrm{min} / 1.73 \mathrm{~m}^{2}, P=0.005$, respectively). In non-AKI patients with baseline eGFR $\geq 60 \mathrm{~mL} / \mathrm{min} / 1.73 \mathrm{~m}^{2}$, there was a modest decrease in eGFR at 6 month (mean difference $-4.0 ; 95 \% \mathrm{CI}-6.4,-1.6 \mathrm{ml} / \mathrm{min} / 1.73 \mathrm{~m}^{2}$, $P=0.001)$. Conversely, in non-AKI patients with eGFR $30-59$ and $<30 \mathrm{~mL} / \mathrm{min} / 1.73 \mathrm{~m}^{2}$, there was an increase in eGFR at 6 months (mean difference 2.4; 95\% CI 0.8, $2.4 \mathrm{~mL} / \mathrm{min} / 1.73 \mathrm{~m}^{2}$; $P=0.004$ and $5.3 ; 95 \%$ CI $2.8,7.8 \mathrm{~mL} / \mathrm{min} / 1.73 \mathrm{~m}^{2} ; P=0.001$, respectively).

Conclusion: In patients undergoing TAVR, change in renal function is significantly related to pre-procedural kidney function. AKI significantly impacts renal function at six months post TAVR. CKD patients who do not develop AKI, may benefit from TAVR by an increase in eGFR at six months.
\end{abstract}

\section{Implication for health policy/practice/research/medical education}

Impact of transcatheter aortic valve replacement (TAVR) on renal function especially in patients with chronic kidney disease (CKD) is unclear. In this current study, we investigated the change in eGFR following TAVR in non-advanced CKD patients with severe aortic stenosis (AS) stratified by levels of eGFR $\left(\geq 60,30-59\right.$, and $\left.<30 \mathrm{~mL} / \mathrm{min} / 1.73 \mathrm{~m}^{2}\right)$. Our findings were as follows; 1) acute kidney injury (AKI) significantly decreased eGFR at 6 months after TAVR and 2) In patients with no AKI after TAVR, individuals with CKD (particularly eGFR $<30 \mathrm{~mL} / \mathrm{min} / 1.73 \mathrm{~m}^{2}$ ) had a significant increase in eGFR (decrease in SCr) at $6 \mathrm{months}$. Please cite this paper as: Thongprayoon C, Cheungpasitporn W, Kittanamongkolchai W, Srivali N, Greason KL, Kashani KB. Changes in kidney function among patients undergoing transcatheter aortic valve replacement. J Renal Inj Prev. 2017;6(3):216221. DOI: $10.15171 /$ jrip.2017.41.

\section{Introduction}

Transcatheter aortic valve replacement (TAVR) has globally expanded in the past decade and now is acknowledged as a standard approach for patients who have severe aortic stenosis (AS) deemed inoperable with high surgical risk for open-heart aortic valve replacement surgery (1-6). In addition, the recently published studies also suggest that patient selection for TAVR is evolving toward treating lower surgical risk patients (7-9). Thus, to date, more than 200000 TAVR procedures have been 
performed worldwide $(3,10)$.

Despite increasing evidence of treating intermediate surgical-risk patients (7-9), many patients elected for TAVR commonly have renal insufficiency $(11,12)$, one of important predictors for acute kidney injury (AKI) development (13). In addition, AKI following TAVR is very prevalent, varying from $15 \%$ up to $57 \%(10,11,14$ 16). Although patients with chronic kidney disease (CKD) carry a higher risk of developing AKI, improvement in estimated glomerular filtration rate (eGFR) following surgical aortic valve replacements (SAVRs) has been demonstrated in patients with CKD after relief of severe aortic valve diseases $(17,18)$.

\section{Objectives}

Little is known regarding the impact of TAVR on renal function especially in patients with CKD. Thus, we conducted this retrospective study to evaluate the change in eGFR after TAVR.

\section{Patients and Methods}

We conducted a retrospective observational study at Mayo Clinic Hospital, a quaternary referral hospital in Rochester, Minnesota. Adult patients (age $\geq 18$ years) with AS, who underwent TAVR between January 1st, 2008 and June 30th, 2014 were enrolled. Exclusion criteria were: (a) patients who had advanced CKD stage 5 (eGFR $<15$ $\left.\mathrm{mL} / \mathrm{min} / 1.73 \mathrm{~m}^{2}\right),(b)$ patients who received dialysis $(\leq 14$ days prior to TAVR), and (c) patients without research authorization.

Clinical, laboratory, pre- and post-procedural data were obtained from our institutional electronic medical record system. The Society of Thoracic Surgeons' (STS) adult cardiac surgery risk score was calculated for each patient as a surrogate for operative mortality risk (19-21). The eGFR was calculated using the CKD epidemiology collaboration equation (22). Primary outcomes were the changes in eGFR after TAVR; at the day of hospital discharge and at 6 months. We stratified patients based on AKI and CKD stages. AKI after TAVR was defined by an increase in serum creatinine (SCr) of $\geq 0.3 \mathrm{mg} / \mathrm{dL}(\geq 26.5$ $\mu \mathrm{mol} / \mathrm{L}$ ) within 48 hours after TAVR or a relative increase of $\geq 50 \%$ ) of the KDIGO definition (23).

\section{Ethical issues}

1) The research followed the tenets of the Declaration of Helsinki; 2) The Institutional Review Board affirmed our study and informed consent for patients with research authorization was waived; and 3) This study was approved by the Ethics Committee of Mayo Clinic.

\section{Statistical analysis}

To identify the differences in clinical characteristics between patients with and without AKI after TAVR, student's $t$ test was used for continuous variables and the chi-squared test, or Fisher's exact test was used for categorical variables, as appropriate. The changes in
eGFR and SCr before and after TAVR were tested using paired $t$ test. A two-sided $P$ value of $<0.05$ was considered statistically significant. All analyses were performed using JMP statistical software version 10 (SAS, Cary, NC).

\section{Results}

A total of 390 TAVR procedures for AS were conducted during the study time period. Three were excluded due to advance CKD stage 5 or receiving dialysis ( $\leq 14$ days before TAVR). One patient had no research authorization and therefore was excluded. A total of 386 patients were included in the analysis.

\section{Patient characteristics}

In our cohort, the mean age was $81 \pm 8$ years, and $56 \%$ were male. Ninty-seven percent of patients were Caucasian. Mean baseline eGFR of patients undergoing TAVR was $55 \pm 21 \mathrm{mLmin} / 1.73 \mathrm{~m}^{2}$. The mean STS adult cardiac surgery risk score was $8.6 \pm 6.3$. Most of the TAVR procedures were performed via transfemoral (51\%), followed by transapical (44\%), and transaortic (5\%) approaches. Table 1 demonstrated baseline characteristics of the enrolled cohort.

Change in kidney function at in patients with or without AKI after TAVR

Of 386 patients undergoing TAVR, 106 (28\%) developed AKI. Overall baseline eGFR was $48 \pm 22 \mathrm{~mL} / \mathrm{min} / 1.73 \mathrm{~m}^{2}$ in patients who developed AKI after TAVR and $58 \pm 19 \mathrm{~mL} /$ $\min / 1.73 \mathrm{~m}^{2}$ in those who did not have AKI. In patients with AKI, there was significant reduction in eGFR at the hospital discharge, which remained significantly reduced at 6 months post TAVR (mean differences $-7.1 ; 95 \% \mathrm{CI}$ $-9.8,-4.3 \mathrm{~mL} / \mathrm{min} / 1.73 \mathrm{~m}^{2}, P<0.001$ and $-4.2 ; 95 \% \mathrm{CI}-7.1$, $-1.3 \mathrm{~mL} / \mathrm{min} / 1.73 \mathrm{~m}^{2}, P=0.005$, respectively), shown in Table 2.

In non-AKI patients with baseline eGFR $\geq 60 \mathrm{~mL} /$ $\mathrm{min} / 1.73 \mathrm{~m}^{2}$, there was a modest decrease in eGFR at 6 month after TAVR (mean difference -4.0 ; 95\% CI -6.4, $\left.-1.6 \mathrm{~mL} / \mathrm{min} / 1.73 \mathrm{~m}^{2}, P=0.001\right)$. Conversely, in non-AKI patients with eGFR $30-59$ and $<30 \mathrm{~mL} / \mathrm{min} / 1.73 \mathrm{~m}^{2}$, there was an increase in eGFR at 6 months (mean difference 2.4; $95 \%$ CI $0.8,4.0 \mathrm{ml} / \mathrm{min} / 1.73 \mathrm{~m}^{2} ; P=0.004$ and $5.3 ; 95 \% \mathrm{CI}$ $2.8,7.8 \mathrm{~mL} / \mathrm{min} / 1.73 \mathrm{~m}^{2} ; P=0.001$, respectively), shown in Table 2.

Change in SCr after TAVR was also shown in Table 3. Consistent with change in eGFR after TAVR, in patients with AKI, there was significant increase in SCr at the hospital discharge, which remained significantly elevated at 6 months after TAVR (mean differences 0.27; 95\% CI $0.18,0.36 \mathrm{mg} / \mathrm{dL}, P<0.001$ and $0.17 ; 95 \%$ CI $0.07,0.26$ $\mathrm{mg} / \mathrm{dL}, P=0.001$, respectively). In non-AKI patients with baseline eGFR $\geq 60 \mathrm{~mL} / \mathrm{min} / 1.73 \mathrm{~m}^{2}$, there was a modest increase in SCr at 6 months after TAVR (mean differences 0.06; 95\% CI 0.02, $0.09 \mathrm{mg} / \mathrm{dL}, P=0.001)$. Conversely, in non-AKI patients with eGFR $<30 \mathrm{~mL} / \mathrm{min} / 1.73 \mathrm{~m}^{2}$, there was a decrease in SCr at 6 months (mean differences -0.25; 
Table 1. Clinical characteristics and outcomes of TAVR

\begin{tabular}{|c|c|c|c|c|}
\hline Characteristics & All $(n=386)$ & AKI $(n=106)$ & No AKI $(n=280)$ & $P$ value \\
\hline STS risk score ${ }^{a}$ & $8.6 \pm 6.3$ & $9.7 \pm 6.0$ & $8.1 \pm 6.4$ & 0.02 \\
\hline Age (year) ${ }^{a}$ & $81 \pm 8$ & $82 \pm 7$ & $81 \pm 8$ & 0.22 \\
\hline Male sex ${ }^{b}$ & $217(56)$ & $62(58)$ & $155(55)$ & 0.58 \\
\hline White ${ }^{b}$ & $374(97)$ & $101(95)$ & $273(98)$ & 0.26 \\
\hline Body Mass Index $\left(\mathrm{kg} / \mathrm{m}^{2}\right)^{\mathrm{a}}$ & $30.4 \pm 7.5$ & $30.3 \pm 7.0$ & $30.4 \pm 7.6$ & 0.96 \\
\hline Baseline eGFR $\left(\mathrm{mL} / \mathrm{min} / 1.73 \mathrm{~m}^{2}\right)^{a}$ & $55 \pm 21$ & $48 \pm 22$ & $58 \pm 19$ & $<0.001$ \\
\hline NYHA class III-IV b & $335(87)$ & $97(92)$ & $238(85)$ & 0.09 \\
\hline \multicolumn{5}{|l|}{ Comorbidity } \\
\hline Diabetes mellitus ${ }^{b}$ & $157(41)$ & $51(48)$ & $106(38)$ & 0.07 \\
\hline Hypertension ${ }^{b}$ & $349(90)$ & $100(94)$ & $249(89)$ & 0.11 \\
\hline Dyslipidemia $^{\mathrm{b}}$ & $346(90)$ & $98(92)$ & $248(89)$ & 0.26 \\
\hline Myocardial infarction $^{b}$ & $139(36)$ & $41(39)$ & $98(35)$ & 0.50 \\
\hline Congestive heart failure ${ }^{b}$ & $223(58)$ & $68(64)$ & $55(155)$ & 0.12 \\
\hline Stroke ${ }^{b}$ & $110(28)$ & $30(28)$ & $80(29)$ & 0.96 \\
\hline Peripheral vascular disease ${ }^{b}$ & $226(59)$ & $68(64)$ & $158(56)$ & 0.17 \\
\hline Anemiab $^{b}$ & $10(3)$ & $4(4)$ & $6(2)$ & 0.37 \\
\hline Chronic lung disease $^{b}$ & $240(62)$ & $64(60)$ & $176(63)$ & 0.65 \\
\hline Smoking within 1 year ${ }^{b}$ & $11(3)$ & $3(3)$ & $8(3)$ & 0.99 \\
\hline \multicolumn{5}{|l|}{ Prior cardiac intervention } \\
\hline $\mathrm{PCl}^{\mathrm{b}}$ & $199(52)$ & $61(58)$ & $138(49)$ & 0.15 \\
\hline Cardiac surgery ${ }^{\mathrm{b}}$ & $181(47)$ & $44(42)$ & $137(49)$ & 0.19 \\
\hline $\mathrm{CABG}^{\mathrm{b}}$ & $167(43)$ & $43(41)$ & $124(44)$ & 0.51 \\
\hline Valve surgery ${ }^{b}$ & $84(22)$ & $28(26)$ & $56(20)$ & 0.17 \\
\hline Aortic valve surgery ${ }^{b}$ & $10(3)$ & $1(1)$ & $9(3)$ & 0.21 \\
\hline \multicolumn{5}{|l|}{ Echocardiographic finding } \\
\hline Ejection fraction ${ }^{a}$ & $56 \pm 13$ & $54 \pm 13$ & $57 \pm 13$ & 0.08 \\
\hline Aortic valve gradient ${ }^{\mathrm{a}}$ & $48 \pm 14$ & $46 \pm 14$ & $49 \pm 14$ & 0.07 \\
\hline Aortic valve insufficiency ${ }^{b}$ & $209(54)$ & $58(55)$ & $151(54)$ & 0.89 \\
\hline Mitral valve dysfunction ${ }^{b}$ & $300(78)$ & $81(76)$ & $219(78)$ & 0.70 \\
\hline \multicolumn{5}{|l|}{ Preoperative medication } \\
\hline $\mathrm{ACEl} / \mathrm{ARB}^{\mathrm{b}}$ & $157(41)$ & $42(40)$ & $116(41)$ & 0.75 \\
\hline Beta-blocker ${ }^{b}$ & $266(69)$ & $72(68)$ & $194(69)$ & 0.80 \\
\hline Statin ${ }^{b}$ & $281(73)$ & $74(70)$ & $207(74)$ & 0.42 \\
\hline Aspirin $^{b}$ & $284(74)$ & $71(67)$ & $213(76)$ & 0.07 \\
\hline Normal sinus rhythm ${ }^{b}$ & $280(73)$ & $71(67)$ & $209(75)$ & 0.13 \\
\hline Elective surgery ${ }^{b}$ & $368(96)$ & $98(92)$ & $270(96)$ & 0.10 \\
\hline Arterial approach & & & & $<.001$ \\
\hline Transfemoral $^{b}$ & $195(51)$ & $36(34)$ & $159(57)$ & \\
\hline Transapical $^{b}$ & $171(44)$ & $65(61)$ & $106(38)$ & \\
\hline Transaortic $^{b}$ & $20(5)$ & $5(5)$ & $15(5)$ & \\
\hline Surgery duration $(\mathrm{min})^{a}$ & $128 \pm 52$ & $132 \pm 63$ & $126 \pm 47$ & 0.39 \\
\hline RBC transfusion needed ${ }^{b}$ & $129(33)$ & $48(45)$ & $81(29)$ & 0.002 \\
\hline Contrast amount $(\mathrm{mL})^{\mathrm{a}}$ & $94 \pm 56$ & $96 \pm 57$ & $93 \pm 55$ & 0.32 \\
\hline Intra-aortic balloon pump ${ }^{b}$ & $7(2)$ & $5(5)$ & $2(1)$ & 0.02 \\
\hline
\end{tabular}

${ }^{a}$ Continuous variables are reported as mean \pm standard deviation; ${ }^{b}$ Categorical variables are reported as count (percentage).

Abbreviations: ACEI, angiotensin-converting enzyme inhibitor; AKI, acute kidney injury; ARB, angiotensin II receptor blocker; CABG, coronary artery bypass graft surgery; eGFR, estimated glomerular filtration rate; NYHA, New York Heart Association; PCI, percutaneous coronary intervention; RBC, red blood cell; STS, Society of Thoracic Surgeons; TAVR, transcatheter aortic valve replacement.

95\% CI $-0.36,-0.14 \mathrm{mg} / \mathrm{dL}, P<0.001)$.

\section{Discussion}

Previous studies have demonstrated an association between $\mathrm{CKD}$ and poor outcomes in patients undergoing TAVR $(24,25)$. However, data on the change of renal function after relief of severe AS by TAVR are limited. In the current study, we examined the change in eGFR following TAVR in non-advanced CKD patients with severe AS stratified by levels of eGFR $(\geq 60,30-59$, and $<30$ $\mathrm{mL} / \mathrm{min} / 1.73 \mathrm{~m}^{2}$ ). Our findings were as follows; 1) AKI significantly decreased eGFR at six months after TAVR and 2) In patients with no AKI after TAVR, individuals with CKD (particularly eGFR $<30 \mathrm{~mL} / \mathrm{min} / 1.73 \mathrm{~m}^{2}$ ) had a significant increase in eGFR (decrease in SCr) at 6 months. Although the precise underlying mechanisms of recovery of kidney function among CKD patients after TAVR is still unclear, we believe that the increase in eGFR is likely 
Table 2. Change in eGFR at hospital discharge and 6 months after TAVR

\begin{tabular}{|c|c|c|c|c|c|c|c|}
\hline Group & Baseline & Hospital discharge & $\begin{array}{c}\text { Mean difference } \\
(95 \% \mathrm{Cl})\end{array}$ & $P$ value & $\begin{array}{c}6 \text { months after } \\
\text { TAVR }\end{array}$ & $\begin{array}{c}\text { Mean difference }(95 \% \\
\mathrm{CI})\end{array}$ & $P$ value \\
\hline All & $55 \pm 21$ & $55 \pm 22$ & $0.2(-1.0,1.4)$ & 0.78 & $54 \pm 21$ & $-1.4(-2.7,-0.1)$ & 0.03 \\
\hline \multicolumn{8}{|l|}{ GFR group } \\
\hline$\geq 60$ & $77 \pm 11$ & $75 \pm 14$ & $-1.9(-4.0,0.1)$ & 0.07 & $72 \pm 15$ & $-5.0(-7.4,-2.7)$ & $<0.001$ \\
\hline $30-59$ & $44 \pm 8$ & $46 \pm 14$ & $2.0(0.3,3.6)$ & 0.02 & $45 \pm 14$ & $0.8(-0.9,2.4)$ & 0.36 \\
\hline$<30$ & $24 \pm 4$ & $24 \pm 8$ & $-0.4(-2.6,1.8)$ & 0.73 & $27 \pm 9$ & $2.3(-0.2,4.7)$ & 0.06 \\
\hline \multicolumn{8}{|l|}{ AKI status } \\
\hline Non-AKI & $58 \pm 19$ & $61 \pm 20$ & $2.9(1.8,4.1)$ & $<0.001$ & $58 \pm 19$ & $-0.4(-1.8,1.0)$ & 0.62 \\
\hline$\geq 60$ & $76 \pm 11$ & $78 \pm 13$ & $1.3(-0.5,3.1)$ & 0.16 & $72 \pm 14$ & $-4.0(-6.4,-1.6)$ & 0.001 \\
\hline $30-59$ & $45 \pm 8$ & $49 \pm 12$ & $4.3(2.8,5.9)$ & $<0.001$ & $47 \pm 13$ & $2.4(0.8,4.0)$ & 0.004 \\
\hline$<30$ & $26 \pm 3$ & $31 \pm 4$ & $4.1(1.7,6.5)$ & 0.003 & $32 \pm 4$ & $5.3(2.8,7.8)$ & 0.001 \\
\hline AKI & $48 \pm 22$ & $41 \pm 20$ & $-7.1(-9.8,-4.3)$ & $<0.001$ & $43 \pm 22$ & $-4.2(-7.1,-1.3)$ & 0.005 \\
\hline
\end{tabular}

Abbreviations: AKI, acute kidney injury; eGFR, estimated glomerular filtration rate; TAVR, transcatheter aortic valve replacement.

Table 3. Change in SCr at hospital discharge and 6 months after TAVR

\begin{tabular}{|c|c|c|c|c|c|c|c|}
\hline Group & Baseline & Hospital discharge & $\begin{array}{c}\text { Mean difference } \\
(95 \% \mathrm{Cl})\end{array}$ & $P$ value & $\begin{array}{c}6 \text { months after } \\
\text { TAVR }\end{array}$ & $\begin{array}{c}\text { Mean difference }(95 \% \\
\mathrm{Cl})\end{array}$ & $P$ value \\
\hline All & $1.2 \pm 0.5$ & $1.3 \pm 0.6$ & $0.03(0.001,0.07)$ & 0.04 & $1.3 \pm 0.5$ & $0.05(0.02,0.08)$ & 0.004 \\
\hline \multicolumn{8}{|l|}{ GFR group } \\
\hline$\geq 60$ & $0.9 \pm 0.2$ & $0.9 \pm 0.2$ & $0.03(-0.001,0.05)$ & 0.06 & $0.9 \pm 0.2$ & $0.07(0.04,0.11)$ & $<0.001$ \\
\hline $30-59$ & $1.4 \pm 0.3$ & $1.4 \pm 0.4$ & $0.01(-0.04,0.06)$ & 0.69 & $1.4 \pm 0.4$ & $0.04(-0.01,0.08)$ & 0.11 \\
\hline$<30$ & $2.1 \pm 0.4$ & $2.3 \pm 0.8$ & $0.19(-0.006,0.39)$ & 0.06 & $2.1 \pm 0.8$ & $-0.003(-0.21,0.20)$ & 0.98 \\
\hline \multicolumn{8}{|l|}{ AKI status } \\
\hline Non-AKI & $1.2 \pm 0.4$ & $1.1 \pm 0.4$ & $-0.06(-0.08,-0.04)$ & $<0.001$ & $1.2 \pm 0.4$ & $0.003(-0.02,0.03)$ & 0.85 \\
\hline$\geq 60$ & $0.9 \pm 0.2$ & $0.8 \pm 0.2$ & $-0.02(-0.04,0.004)$ & 0.10 & $0.9 \pm 0.2$ & $0.06(0.02,0.09)$ & 0.001 \\
\hline $30-59$ & $1.3 \pm 0.3$ & $1.3 \pm 0.3$ & $-0.08(-0.11,0.04)$ & $<0.001$ & $1.3 \pm 0.3$ & $-0.02(-0.06,0.02)$ & 0.23 \\
\hline$<30$ & $2.0 \pm 0.4$ & $1.7 \pm 0.4$ & $-0.19(-0.29,-0.09)$ & 0.001 & $1.6 \pm 0.4$ & $-0.25(-0.36,-0.14)$ & $<0.001$ \\
\hline AKI & $1.4 \pm 0.6$ & $1.7 \pm 0.8$ & $0.27(0.18,0.36)$ & $<0.001$ & $1.6 \pm 0.7$ & $0.17(0.07,0.26)$ & 0.001 \\
\hline
\end{tabular}

Abbreviations: AKI, acute kidney injury; eGFR, estimated glomerular filtration rate; TAVR, transcatheter aortic valve replacement, SCr, serum creatinine.

attributed to an improvement in the cardiac forward flow after relief of severe AS resulting in a higher organ perfusion including kidneys $(17,26)$. Also, improvement in a right heart function can lead to a decrease in renal venous congestion $(17,27)$. As our finding of an increase in eGFR in CKD patients after TAVR, improvement in eGFR following SAVR has been demonstrated $(17,18)$. Thus, improvement in cardiac functions after TAVR likely plays a significant role in an improvement in kidney function. In patients with advanced CKD or dialysis dependence, studies have shown a higher rate of early and late mortality following TAVR $(28,29)$. Despite having poorer outcomes after TAVR compared with non-dialysis patients, TAVR is comparable with SAVR in ESRD patients on dialysis based on a propensity-matched comparison of all Medicare feefor-service patients undergoing TAVR or SAVR (29). Data on renal function change after TAVR in advanced CKD (nondialysis stage $5 \mathrm{CKD}$ ) are limited. Unfortunately, the proportion of nondialysis stage 5 CKD undergoing TAVR at our institution during the study period was very small and thus we did not enroll in our study. Interestingly, a case of reversal of end-stage renal disease in a patient after TAVR was reported (30). Future studies are required to assess renal function change in this high-risk patient population.

\section{Conclusion}

In conclusion, our study demonstrates that change in eGFR is significantly related to baseline kidney function of patients undergoing TAVR. AKI significantly reduces eGFR at 6 months post TAVR. Without AKI after TAVR, patients with CKD, particularly baseline eGFR between 15 and $30 \mathrm{~mL} / \mathrm{min} / 1.73 \mathrm{~m}^{2}$ may benefit from TAVR by an improvement in eGFR at 6 months.

\section{Limitations of the study}

There are several limitations to our study. First, our study has a retrospective observational design and patients in our center are predominantly Caucasian populations, conceivably causing selection bias and restricting the generalizability of our findings. Second, the cause of a slight reduction in eGFR (approximately $-4.0 \mathrm{~mL} / \mathrm{min} / 1.73 \mathrm{~m}^{2}$ ) at 6 months in non-AKI patients with baseline eGFR $\geq$ $60 \mathrm{~mL} / \mathrm{min} / 1.73 \mathrm{~m}^{2}$ is unclear. Calculating eGFR based on SCr has a few limitations $(31,32)$. It is possible that patients after TAVR had improved function status and muscle mass, resulting in higher SCr levels (33). Unfortunately, our data regarding body mass index (BMI) at 6 months follow-up are limited. Future studies with a more accurate assessment of GFR are needed. 
Authors' contribution

CT, WC, WK, and NS performed data acquisition for the cohort, statistical analysis, and participated in initial manuscript creation. KLG and KK conceived of the study, participated in design and coordination, performed data analysis, and drafted and edited the manuscript. KK supervised the project. All authors read and approved the manuscript.

\section{Conflicts of interest}

The authors declare that they have no conflicting interest.

\section{Ethical considerations}

Ethical issues (including plagiarism, data fabrication, double publication) have been completely observed by the authors.

\section{Funding/Support}

None.

\section{References}

1. Tsai MT, Tang GH, Cohen GN. Year in review: transcatheter aortic valve replacement. Curr Opin Cardiol. 2016;31:13947. doi: 10.1097/HCO.0000000000000260.

2. Nishimura RA, Otto CM, Bonow RO, Carabello BA, Erwin JP 3rd, Guyton RA, et al. 2014 AHA/ACC guideline for the management of patients with valvular heart disease: a report of the American College of Cardiology/American Heart Association Task Force on Practice Guidelines. J Thorac Cardiovasc Surg. 2014;148:e1-e132. doi: 10.1161/ CIR.0000000000000029.

3. Saji M, Lim DS. Transcatheter Aortic Valve Replacement in Lower Surgical Risk Patients: Review of Major Trials and Future Perspectives. Curr Cardiol Rep. 2016;18:103. doi: 10.1007/s11886-016-0772-3.

4. Thongprayoon C, Cheungpasitporn W, Srivali N, Harrison AM, Kittanamongkolchai W, Greason KL, et al. Transapical versus transfemoral approach and risk of acute kidney injury following transcatheter aortic valve replacement: a propensity-adjusted analysis. Ren Fail. 2017;39:13-8.

5. Thongprayoon C, Cheungpasitporn W, Gillaspie EA, Greason KL, Kashani KB. Association of blood transfusion with acute kidney injury after transcatheter aortic valve replacement: a meta-analysis. World J Nephrol. 2016;5:482488. doi: 10.5527/wjn.v5.i5.482.

6. Thongprayoon C, Cheungpasitporn W, Gillaspie EA, Greason KL, Kashani KB. The risk of acute kidney injury following transapical versus transfemoral transcatheter aortic valve replacement: a systematic review and metaanalysis. Clin Kidney J. 2016;9:560-566. doi: 10.1093/ckj/ sfw055.

7. Leon MB, Smith CR, Mack MJ, Makkar RR, Svensson LG, Kodali SK, et al. Transcatheter or Surgical aortic-valve replacement in intermediate-risk patients. N Engl J Med. 2016;374:1609-1620. doi: 10.1056/NEJMoa1514616.

8. Tamburino C, Barbanti M, D'Errigo P, Ranucci M, Onorati F, Covello RD, et al. 1-Year outcomes after transfemoral transcatheter or surgical aortic valve replacement: results from the italian OBSERVANT study. J Am Coll Cardiol. 2015;66:804-12. doi: 10.1016/j.jacc.2015.06.013.

9. Thyregod HG, Steinbruchel DA, Ihlemann N, Nissen H,
Kjeldsen BJ, Petursson P, et al. Transcatheter versus surgical aortic valve replacement in patients with severe aortic valve stenosis: 1-year results from the all-comers NOTION randomized clinical trial. J Am Coll Cardiol 2015;65:218494. doi: 10.1016/j.jacc.2015.03.014.

10. Cheungpasitporn W, Thongprayoon C, Kashani K. Transcatheter aortic valve replacement: a kidney's perspective. J Renal Inj Prev. 2016;5:1-7. 10.15171/ jrip.2016.01.

11. Najjar M, Salna M, George I. Acute kidney injury after aortic valve replacement: incidence, risk factors and outcomes. Expert Rev Cardiovasc Ther. 2015;13:301-16. doi: $\quad 10.1586 / 14779072.2015 .1002467$.

12. Thongprayoon C, Cheungpasitporn W, Srivali N, Kittanamongkolchai W, Greason KL, Kashani KB. Incidence and risk factors of acute kidney injury following transcatheter aortic valve replacement. Nephrology (Carlton). 2016;21:1041-6. doi:10.1111/nep.12704

13. Singh P, Rifkin DE, Blantz RC. Chronic kidney disease: an inherent risk factor for acute kidney injury? Clin J Am Soc Nephrol. 2010;5:1690-5. doi: 10.2215/CJN.00830110.

14. Thongprayoon C, Cheungpasitporn W, Srivali N, Ungprasert P, Kittanamongkolchai W, Greason KL, et al. Acute kidney injury after transcatheter aortic valve replacement: a systematic review and meta-analysis. Am J Nephrol. 2015;41:372-82. doi: 10.1159/000431337.

15. Thongprayoon C, Cheungpasitporn W, Srivali N, Harrison AM, Gunderson TM, Kittanamongkolchai W, et al. AKI after transcatheter or surgical aortic valve replacement. J Am Soc Nephrol. 2016;27:1854-60. doi: 10.1681/ASN.2015050577.

16. Thongprayoon C, Cheungpasitporn W, Podboy AJ, Gillaspie EA, Greason KL, Kashani KB. The effects of contrast media volume on acute kidney injury after transcatheter aortic valve replacement: a systematic review and meta-analysis. J Evid Based Med. 2016. doi: 10.1111/jebm.12208.

17. Najjar M, Yerebakan H, Sorabella RA, Guglielmetti L, Vandenberge J, Kurlansky P, et al. Reversibility of chronic kidney disease and outcomes following aortic valve replacementdagger. Interact Cardiovasc Thorac Surg. 2015;21:499-505. doi: 10.1093/icvts/ivv196.

18. Doganci S, Durgun B, Kadan M, Yildirim V. eComment. It is difficult to clearly explain the effect of aortic valve replacement on the reversibility of chronic kidney disease. Interact Cardiovasc Thorac Surg. 2015;21:505. doi: 10.1093/ icvts/ivv246.

19. Shahian DM, Edwards FH. The Society of Thoracic Surgeons 2008 cardiac surgery risk models: introduction. Ann Thorac Surg. 2009;88:S1.

20. O'Brien SM, Shahian DM, Filardo G, Ferraris VA, Haan CK, Rich JB, et al. The Society of Thoracic Surgeons 2008 cardiac surgery risk models: part 2--isolated valve surgery. Ann Thorac Surg. 2009;88:S23-42.

21. Shahian DM, O’Brien SM, Filardo G, Ferraris VA, Haan CK, Rich JB, et al. The Society of Thoracic Surgeons 2008 cardiac surgery risk models: part 3--valve plus coronary artery bypass grafting surgery. Ann Thorac Surg. 2009;88:S43-62.

22. Levey AS, Stevens LA, Schmid CH, Zhang YL, Castro AF 3rd, Feldman HI, et al. A new equation to estimate glomerular filtration rate. Ann Intern Med. 2009;150:60412.

23. Kellum JA, Lameire N. Diagnosis, evaluation, and management of acute kidney injury: a KDIGO summary (Part 1). Crit Care 2013;17:204. 
24. Yamamoto $M$, Hayashida $K$, Mouillet $G$, Hovasse $T$, Chevalier B, Oguri A, et al. Prognostic value of chronic kidney disease after transcatheter aortic valve implantation. J Am Coll Cardiol. 2013;62:869-77. doi: 10.1016/j. jacc.2013.04.057.

25. Arnold SV, Reynolds MR, Lei Y, Magnuson EA, Kirtane AJ, Kodali SK, et al. Predictors of poor outcomes after transcatheter aortic valve replacement: results from the PARTNER (Placement of Aortic Transcatheter Valve) trial. Circulation. 2014;129:2682-90. doi: 10.1161/ CIRCULATIONAHA.113.007477.

26. Keleş T, Ayhan H, Durmaz T, Sari C, Aslan AN, Erdogan $\mathrm{KE}$, et al. Improvement in renal functions with transcatheter aortic valve implantation. J Geriatr Cardiol. 2013;10:31722. doi: 10.3969/j.issn.1671-5411.2013.04.010.

27. Ahmed A, Campbell RC. Epidemiology of chronic kidney disease in heart failure. Heart Fail Clin. 2008;4:387-99.

28. Allende R, Webb JG, Munoz-Garcia AJ, de Jaegere P, Tamburino C, Dager AE, et al. Advanced chronic kidney disease in patients undergoing transcatheter aortic valve implantation: insights on clinical outcomes and prognostic markers from a large cohort of patients. Eur Heart J. 2014;35:2685-96. doi: 10.1093/eurheartj/ehu175.
29. Kobrin DM, McCarthy FH, Herrmann HC, Anwaruddin S, Kobrin S, Szeto WY, et al. Transcatheter and surgical aortic valve replacement in dialysis patients: a propensitymatched comparison. Ann Thorac Surg. 2015;100:1230-6. doi: 10.1016/j.athoracsur.2015.05.039.

30. Damluji A, Bilsker MS, Cohen MG. Reversal of end stage renal disease in patient with transcatheter aortic valve replacement. Catheter Cardiovasc Interv. 2014;83:482-4. doi: $10.1002 /$ ccd.25127.

31. Thongprayoon C, Cheungpasitporn W, Kittanamongkolchai W, Srivali N, Ungprasert P, Kashani K. Optimum methodology for estimating baseline serum creatinine for the acute kidney injury classification. Nephrology (Carlton). 2015;20:881-6. doi: 10.1007/s40620-015-0211-3.

32. Thongprayoon C, Cheungpasitporn W, Srivali N, Ungprasert P, Kittanamongkolchai W, Kashani K. The impact of fluid balance on diagnosis, staging and prediction of mortality in critically ill patients with acute kidney injury. J Nephrol. 2016;29:221-7.

33. Thongprayoon C, Cheungpasitporn W, Kashani K. Serum creatinine level, a surrogate of muscle mass, predicts mortality in critically ill patients. J Thorac Dis. 2016;8:E305311. doi: $10.21037 /$ jtd.2016.03.62.

Copyright (c) 2017 The Author(s); Published by Nickan Research Institute. This is an open-access article distributed under the terms of the Creative Commons Attribution License (http://creativecommons.org/licenses/by/4.0), which permits unrestricted use, distribution, and reproduction in any medium, provided the original work is properly cited. 